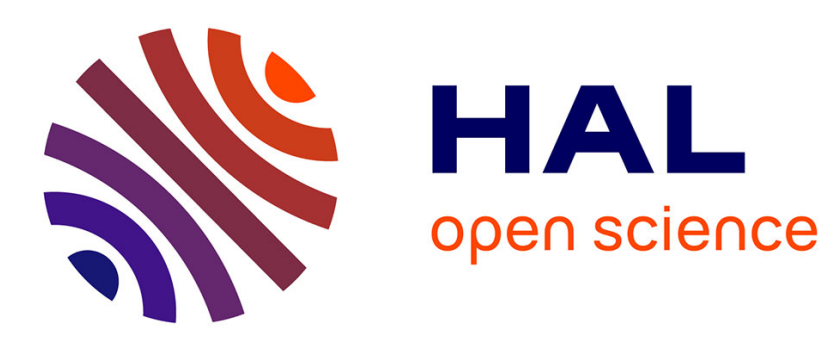

\title{
Interpolated versus Polytopic Gain Scheduling Control Laws for Fin/Rudder Roll Stabilisation of Ships
}

\author{
Vinciane Chéreau, Hervé Tanguy, Guy Lebret
}

\section{To cite this version:}

Vinciane Chéreau, Hervé Tanguy, Guy Lebret. Interpolated versus Polytopic Gain Scheduling Control Laws for Fin/Rudder Roll Stabilisation of Ships. 44th IEEE Conference on Decision and Control, 2005 and 2005 European Control Conference. CDC-ECC '05., Dec 2005, Séville, Spain. pp.2284 - 2289. hal-00369636

\section{HAL Id: hal-00369636 \\ https://hal.science/hal-00369636}

Submitted on 20 Mar 2009

HAL is a multi-disciplinary open access archive for the deposit and dissemination of scientific research documents, whether they are published or not. The documents may come from teaching and research institutions in France or abroad, or from public or private research centers.
L'archive ouverte pluridisciplinaire HAL, est destinée au dépôt et à la diffusion de documents scientifiques de niveau recherche, publiés ou non, émanant des établissements d'enseignement et de recherche français ou étrangers, des laboratoires publics ou privés. 


\title{
Interpolated versus Polytopic Gain Scheduling Control Laws for Fin/Rudder Roll Stabilisation of Ships
}

\author{
Vinciane Chéreau, Hervé Tanguy and Guy Lebret
}

\begin{abstract}
Taking into account the variations of the environment of ships is a means of improving performances of roll stabilisation systems; this can be done through the use of gain-scheduling (GS) control law. In this study, a GS-control law is obtained by interpolation of fixed $\mathbf{H}_{\infty}$ controllers which have been synthetized for different sailing conditions of the ship represented by linear models. The GS controller depends on the ship speed and on a stabilisation quality factor. It is compared to a previously synthetized $H_{\infty}$ LPV controller (Linear Parameters Varying). Simulation results are given.
\end{abstract}

\section{INTRODUCTION}

The behaviour of a ship is greatly influenced by its environment and particulary by the swell. In the domain of ship control, a major improvement in the roll stabilisation systems performances should be to adapt to these environmental conditions: waves, ship speed or loading conditions ...

However, most of the really implemented controllers are independent of these conditions, even if the dependance on the ship speed has been described and used for many years for PID and $\mathrm{H}_{\infty}$ control laws [1], [2]. Also, manual mechanism to cope with changes in the sea state has been introduced [3].

This text is a contribution to building a methodology to obtain parameter dependent control laws for roll stabilisation. A case study is described where two varying parameters are introduced: the ship speed and a desired stabilisation quality factor.

Gain scheduling is a way to obtain parameter dependent controllers. Classical designs [2] consist in interpolating several invariant controllers tuned for different operating points of the plant or to switch controllers when sailing conditions change. Another possibility, is to directly synthetize a varying parameter controller from a known parameter-dependent model of the plant. Both approaches have theirs pros\&cons. The second approach has already been investigated in [4]. In [4] a four steps methodology to obtain a $\mathrm{H}_{\infty} / \mathrm{LMI}$ gain scheduling control law was presented. It was based on polytopic representation of a Linear Parameter Varying (LPV) model of a ship .

The first approach, more popular in industrial applications, is explored here. Simulation comparisons of performances of both controllers are shown in the case study. It is a frigate type ship (length $120 \mathrm{~m}$, displacement 3000 metric tons); the

V. Chereau is with IREENA ( www.polytech.univ-nantes.fr/ireena ), Nantes, France, misschereau@netcourrier.com

H. Tanguy is with Sirehna ( www.sirehna.com ), Nantes, France sirehnaesirehna.ec-nantes.fr

G. Lebret is with IRCCyN ( www.irccyn.ec-nantes.fr ), Ecole Centrale de Nantes, France, guy.lebreteirccyn.ec-nantes.fr considered environmental conditions are sea state 5; the load is considered constant.

The paper is organized as follows: in section II, a short review of Gain Scheduling (GS) controllers is given. In section III, the context [4] of the study is described to finally detail the computation of the interpolated GS-controller in section IV. The simulated performances of the study case are described in section V. Section VI is devoted to the conclusion

\section{GAIN SCHEDULING CONTROL LAW}

Gain scheduling is one of the popular approaches to nonlinear control design [5], [6]; it has been widely and successfully applied especially in aerospace for the control of airplane or missile. The synthesis model of the nonlinear system can be a set of linear representations corresponding to different behaviours or operating points of the system, parameterized by a appropriate measurable quantity $\theta$. It can also directly be a LPV model in $\theta$. The general form of the synthetized controller, parameterized by the same quantity, is nonlinear, but it is linear for fixed value of $\theta$. In few words, one synthetizes a parameterized controller for a representation of the process parameterized by the same measurable quantity.

\section{A. Classical Gain-Scheduling design}

In this approach the controller is generally obtained in three steps [5]:

- Operating points of the system are parameterized by a varying quantity $\theta$ and the dynamics are locally approximated by linearisation.

- Linear time invariant controllers are designed for each operating point.

- A nonlinear controller is built based on the set of linear controllers.

Among the possible realisations of the nonlinear controller, interpolation is possible if all the linear controllers have compatible structure which permit smooth interpolation between the designs. In practice, ad hoc (depending on the context) interpolation schemes appear to be commonly employed [5]. It can be the interpolation of transfer functions, or of the matrices of state representations; it can also be the interpolation of the zeros, poles and gain of the controllers transfer functions [7].

These simple, attractive schemes have the drawback that, in most cases, no theoretical proof of the closed loop stability is assured; However, it has often proven to be very efficient in practice and sufficient for an engineering point of view. 
The aim of the present study is to obtain such a controller in the case study of the roll stabilisation of the frigate type ship and compare it with a controller synthetized in [4] with the following approach.

\section{B. LPV Gain-Scheduling}

If a LPV approximation of the nonlinear system exists (which is perhaps not possible) or if this is a natural acceptable representation of the system, there exists LMI techniques to design $\mathrm{H}_{2}$ or $\mathrm{H}_{\infty}$ controllers which are generalizations of the LMI techniques for LTI systems [8], [9].

The main advantages of this approach is that, in this case, the stability is assured. However the generalizations of the LTI results are possible only with particular representations of the system: affine, polytopic or LFT (Linear Fractional Transformation) representations. Unfortunately, these reformulations of the system model introduce conservatism. The polytopic representation especially defines a greater set of systems to control than actually needed. Moreover the synthesis itself brings conservatism.

\section{CONTEXT OF THE STUDY}

In [4], a methodology to obtain a gain scheduling control law for fin/rudder roll stabilisation of ships was described and applied on a particular frigate type ship model (simulation). The techniques used there belong to the LPV gain scheduling approach, using $\mathrm{H}_{\infty}$ and polytopic techniques. This choice was favoured for two main reasons: the will to assure stability and mainly the idea to build a straightforward methodology to obtain a controller for a given ship.

The superiority of the computed $\mathrm{H}_{\infty}$-LPV-GS controller over only one fixed (parameter invariant) $\mathrm{H}_{\infty}$ controller has been shown on simulations. But for a given configuration (i.e. operating point), the performances of the $\mathrm{H}_{\infty}$-LPV-GS controller are worse than those of a fixed $\mathrm{H}_{\infty}$ controller tuned for this configuration (simulations, [10]). This illustrates the conservatism of the approach.

This last point justifies the present study which goal is to obtain an $\mathrm{H}_{\infty}$ interpolated gain scheduling controller $\left(\mathrm{H}_{\infty}\right.$ I-GS) of several fixed $\mathrm{H}_{\infty}$ controllers to evaluate the gain in conservatism.

In the remainder part of this section the model of the process and some needed details of the methodology to obtain the $\mathrm{H}_{\infty}$-LPV-GS controller are briefly recalled [10],[4].

\section{A. Model}

The aim of this section is to show that a ship in a seaway can be modelled as a linear parameter varying system. For a deeper insight see [1], [4], [11] and their references.

Comprehensive models derived from hydrodynamics are too complex to be used in control. Thus, classical acceptable simplifying assumptions are made: amplitude of motions are small; the ship dynamics is independent of the swell frequency. Eventually, the roll motion is considered to be the superposition of the motions induced by the waves and the motions induced by the actuators. This is actually possible by the assumptions that the ship dynamics is linear (see figure
1 for a schematic description). With these assumptions the ship will be modelled as a LPV system (these assumptions are classical, but should be restricted to cases when encounter period (see eq. 2) is not too large).

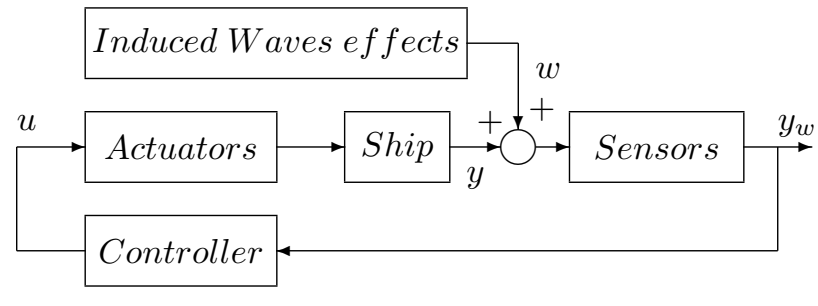

Fig. 1. Control model with output disturbance.

1) Sea disturbance: Complex sea states may be considered to be the superposition of an infinite number of monochromatic waves, distributed in all directions but considered here to be long crested. The frequential content is described by a sea state spectrum. The Bretschneider spectrum (1) will be used in the simulations, with parameters $H_{s}$ (wave height) and $T_{z}$ (mean zero-crossing period) [11].

$$
S_{W}(\omega)=\frac{4 \pi^{3} H_{s}^{2}}{T_{z}^{4} \omega^{5}} \exp \left(\frac{-16 \pi^{3}}{T_{z}^{4} \omega^{4}}\right)
$$

Also note that due to Doppler like effect, the wave frequency observed from a moving ship $\omega_{e}$ is different from the one $\omega$ seen by a motionless observer. The encounter frequency is given by

$$
\omega_{e}=\omega\left(1-\omega \frac{V}{g} \cos \left(\psi_{e}\right)\right)
$$

where $\psi_{e}$ is the angle between the ship motion direction and the wave propagation direction. $V$ is the ship speed.

2) Simulation model: It is very classical to derive the model of a ship from the the mechanical equation of motion in a body fixed frame [11]

$$
M(\eta) \ddot{\eta}+C(\eta, \dot{\eta}) \dot{\eta}=\tau
$$

where $\eta$ is the position of the center of mass, and $\tau$, the external forces, are reduced to the sum of hydrodynamics efforts $\tau_{H}$ due to the waves and of actuators efforts $\tau_{A}$. With the assumption of small motions the hydrodynamics forces $\tau_{H}$ can be divided into three different types [11]: $\tau_{B}$, the buoyancy efforts (from Archimedes' principle), $\tau_{R}$, the radiation forces, $\tau_{W}$, the incident and diffracted waves forces. In the case study $\tau_{A}$ will be supposed to be generated by fins and rudders.

Precise study of these four resulting forces [10], [11], [12] show that they can be rewritten as a sum of terms proportional to the acceleration $\ddot{\eta}$ (added mass), the velocity $\dot{\eta}$ (added damping) and the position $\eta$ (buoyancy) and a term due to the actuators, proportional to their position. Eventually, the introduced coefficients are function of $V$ and $\omega_{e}$ (or $V, \omega$ and $\psi_{e}$ ). A final model is

$$
\begin{array}{r}
\bar{M}\left(\omega, \psi_{e}, V\right) \ddot{\eta}+\bar{D}\left(\omega, \psi_{e}, V\right) \dot{\eta}+G \eta=\ldots \\
F\left(V^{2}\right)[\alpha \delta]^{\top}+\tau_{W}\left(\omega, H_{s}, T_{z}\right)
\end{array}
$$


where $\alpha$ and $\delta$ are respectively the position of the fins and the rudders. $\tau_{W}$ can be simulated from the wave amplitude spectrum (1) and the ship hydrodynamical datas (RAO : Response Amplitude Operators).

If one adds the assumptions that the encounter angle of the swell is constant, that the load is known and invariant, that the ship essentially reacts at its own resonant frequency (the ship dynamics is independent of the swell frequency) a simplified linear model appears. Indeed, the $\bar{M}, \bar{D}$ terms lose their dependency on $\omega$ and $\psi_{e}$, and the final model $\left(\tau_{W\left(H_{s}, T_{z}\right)}\right.$ is set apart) can be rewritten as a linear one with varying parameters in $V$ only. The action of $\tau_{W\left(H_{s}, T_{z}\right)}$ creates disturbance motions. With the assumption that the roll motion is the superposition of the motions induced by the waves and the motions induced by the actuators, the actions of $\tau_{W\left(H_{s}, T_{z}\right)}$ can be simulated as disturbance motions $w_{\left(H_{s}, T_{z}\right)}$ added on the output (figure 1).

The final simulation model is Linear with Varying Parameters (LPV) in $V$ with a perturbation on the output depending on the sea state characterized by $H_{s}$ and $T_{z}$ [10]

$$
\begin{aligned}
\dot{x} & =A(V) x+B u \\
y_{w} & =C x+w_{\left(H_{s}, T_{z}\right)}
\end{aligned}
$$

It is written as a state space model with state $x=$ $[v, p, r, \phi, \psi, \alpha, \dot{\alpha}, \delta, \dot{\delta}]^{T}$ where $v, p, r$ are respectively the sway, roll and yaw velocity; $\phi$ and $\psi$ are the roll and yaw angles. In the case study, $\alpha$ and $\delta$ are the actual position of the actuators (fins and rudders); a second order LTI system has been added to model the actuators dynamics; the control variable, $u$, is the desired position of the actuators; the measures $y_{w}$ considered for control are the perturbed roll velocity $p_{w}$ and yaw angle $\psi_{w}$.

In addition, the simulations take into account the temporal nonlinear aspects of saturation (in angle and rate for both the fins and rudders). A pure delay is also added in temporal simulations to make up for the information transportation effects in the ship internal network.

3) Synthesis Models: The following section describes the methodology introduced in [4] to obtain a $H_{\infty}$-LPV-GS controller. It is a four stage scheme. Its second stage is dedicated to the synthesis of invariant $H_{\infty}$ controllers, for fixed value of $V$, that will also be used in section IV. Each invariant controllers is obtained on the base of a synthesis model derived from the simulation LPV model if $V$ constant and $w=0$. The actuators saturations are not taken into account here.

\section{B. The $H_{\infty}-L P V-G S$-controller}

(5) and (6) show that the motions of the ship depends on its speed $V$, and on the sea state characterized by the parameters $H_{s}$ and $T_{z}$. The idea in [4] has been to synthetize a controller which would be able to adapt to the variation of the ship speed $V$ and which could be able to assure a desired roll attenuation: what has been called Stabilization Quality Factor $S Q F$. Indeed this is a way to adapt to the sea state : with small or medium sea states an increase of the roll attenuation would be obtained by an increase of the stabilization quality factor, with a large sea state $(5,6, \ldots)$ it would probably be necessary to decrease this quality in order to avoid saturations of the actuators. Its value is intended to be directly tuned from the bridge or by an adaption process, taking into account sea state measurements or estimations but also power consumption and actuators saturation levels.

Naturally, others constraints had to be added to synthetize controllers. the following subsection gives an idea of them.

1) The general control specifications [4]: Specifications characterizing the desired behavior of the ship have been chosen from mechanics and passengers' comfort matters:

- reduce the roll motion inside the roll bandwidth and do not amplify it outside,

- keep the yaw angle as constant as possible,

- do not use too much power,

- respect a given power repartition on the actuators. The fins are used only for roll stabilisation. The rudders have a great influence on roll motions, but are primary used to control the yaw,

- tolerate only "acceptable" position and speed saturation of the actuators.

- the closed loop and the controller must be stable,

- some robustness properties are necessary against uncertainties (delay, discretisation...).

2) The four step methodology: In order to derive a gain scheduled controller $K(V, S Q F)(s)$ from $\mathrm{H}_{\infty} / \mathrm{LMI}$ techniques, one needed a LPV standard model defined from the dynamics of the ship and weight functions, translations of the previous specifications. The dynamics of the ship dependent on $V$ (5). The main point here was to introduce the $S Q F$ parameter through the weights of the standard model. It has been defined by the desired depth of a well of the roll sensibility transfer function between the perturbation and the roll angle. Then to comprehensively tune the weights, find invariant $\mathrm{H}_{\infty}$ and finally the LPV $\mathrm{H}_{\infty}$ controller. It has been proposed to follow a four-stage methodology [4]:

- Stage 1: Choose the parameters values in a grid. This defines a finite set, $\Theta$, of possible values $\hat{\theta}$ of the varying parameters $\theta=(V, S Q F)$. In the case study of [4] and hereafter, the set $\Theta$ is defined by a comprehensive gridding, with steps every 5 knots in speed, from 10 to 25 knots, and every 1 unit in SQF quality, from 2 to 8 for $V=15,20$ and 25 knots (six points only between 2 and 3 for $V=10$ knots).

- Stage 2: For each $\hat{\theta}$ of $\Theta$, determine the weights for the standard model that result in a $\mathrm{H}_{\infty}$ controller such that specifications are fulfilled. This tuning of the weights has been realized through the definition of a multiobjective optimisation problem (details in [4] and [13]). Note that there are two optimizations schemes here. The multi-objective optimisation uses the simulation model to tune the weights, while each $\mathrm{H}_{\infty}$ optimisation is based on a synthesis model (section III-A.3) and the tuned weights. Also, note that the final $\mathrm{H}_{\infty}$ LTI controllers will not be exploited in the next stage of this methodology. Instead, this is the set of standard 
models, defined for each $\hat{\theta} \in \Theta$, that will be used to obtain a LPV model.

- Stage 3: Compute a linear standard model with varying parameters $\theta$, from the fixed standard models resulting from stage 2 .

- Stage 4: Compute a gain scheduled controller for the linear varying parameters model (there are functions of commercial toolbox which do that).

3) Application on the case study: This methodology has been applied on a frigate (length $120 \mathrm{~m}$, displacement 3000 metric tons). The considered environmental conditions are sea state 5 for a encounter angle $\psi_{e}=90 \mathrm{deg}$. The load is considered constant.

The superiority of the computed $\mathrm{H}_{\infty}$-LPV-GS controller over a fixed (parameter invariant) $\mathrm{H}_{\infty}$ controller has been shown on simulations [4]. But for a given configuration (or operating point), the performances of the $\mathrm{H}_{\infty}$-LPVGS controller are not as good as the ones of a fixed $\mathrm{H}_{\infty}$ controller especially tuned for this configuration [10].

This explains the motivation for the search of an other controller by interpolation of the $\mathrm{H}_{\infty}$ controllers computed with the previous methodology but not exploited there.

\section{THE $\mathrm{H}_{\infty}$-I-GS CONTROLLER}

\section{A. The family of $H_{\infty}$ LTI controller}

For each value $\hat{\theta}$ of the parameters in $\Theta$, a $\mathrm{H}_{\infty}$ LTI controller $(K(\hat{\theta})(s), \hat{\theta} \in \Theta)$ has been computed in stage 2 of methodology. Each of them is a 24 states dynamical systems with 2 inputs (the yaw angle and the roll velocity) and 2 outputs, the desired positions of fins and rudders.

\section{B. Some possible interpolation techniques}

Different interpolation techniques are described in the literature. Among them ([14], [7]) there is the interpolation of the coefficients of state space representations, or transfert functions [14]; the interpolation of the zeros, poles and gains of these representations [7]. The techniques developed in [14] have the advantage to assure the stability of the closed loop using smooth commutations between different controllers; but has the drawback to require the storage of a lot of them. On the contrary, the last one has the drawback that stability is not theoretically assured but has the advantage to preserve some structure and above all, to deliver just one parameter varying controller; that is the reason why the following development has been influenced by [7].

Each 2 inputs, 2 outputs controllers $(K(\hat{\theta}), \hat{\theta} \in \Theta)$ has been decomposed into 4 SISO controllers $\left(K_{i j}(\hat{\theta}), i=\right.$ $1,2, j=1,2, \hat{\theta} \in \Theta)$. Interpolations have been achieved on each of them. And finally the construction of the multivariable interpolated GS controller has been done.

\section{Interpolation for each SISO controllers}

Every transfer function of the computed controllers $\left(K_{i j}(\hat{\theta}), i=1,2, j=1,2, \hat{\theta} \in \Theta\right)$ have the same number of poles (24), the same number of zeros (23) and one gain. For each of the four transfer functions of all LTI controllers, these structures have been plotted (see figure 2 for a plot of all the poles, figure 3 is a zoom). Note that the poles are not spread out all over the complex plane.

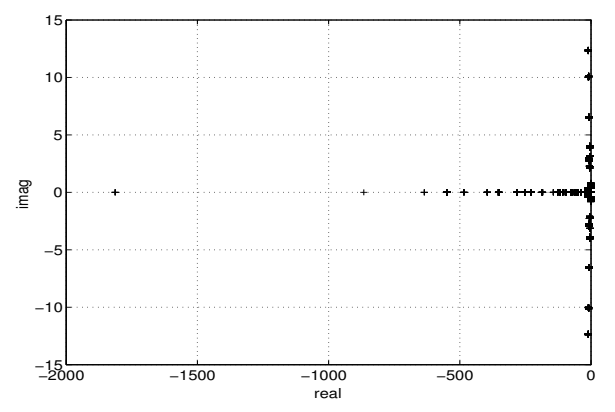

Fig. 2. Position of the poles of $K_{11}(\theta), \theta \in \Theta$.

More precisely, 24 areas of poles and gains, and 23 areas of zeros are easily recognizable; figure 3, a zoom of figure 2 , shows some of these areas.

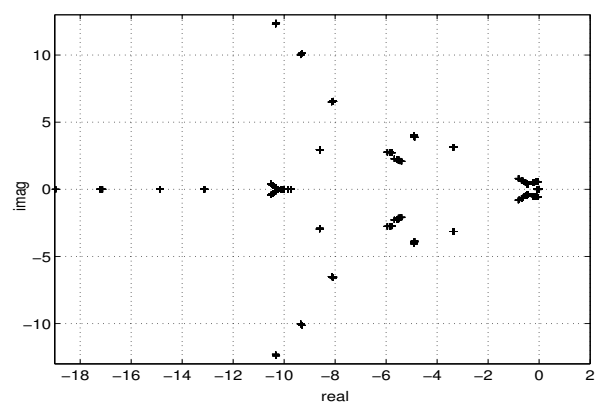

Fig. 3. Zoom of figure 2.

Even better, for the 4 areas of gains, for 23 of 24 areas of poles and for 22 of 23 areas of zeros the migration of points is smooth and not very difficult to determine. For example, figure 4 shows the migration, in a finite area, of one of the 24 poles. On this figure, each straight line corresponds to one of the four possible values of $V$; each points of a line corresponds to a particular value of the SQF parameter.

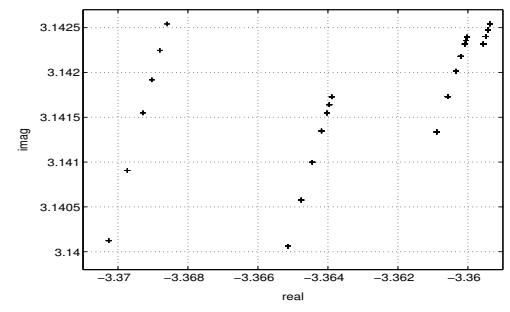

Fig. 4. Migration of one pole depending on $V$ (4 values : 4 straight line) and on $S Q F$ ( 6 or 7 values : 6 or 7 points along each line).

Thus for all these cases (23 poles, 22 zeros and the gains), a first linear interpolation with $\mathrm{SQF}$ as a varying parameter has been done. Two coefficients were necessary for each of them. Then for all the coefficients, a second linear interpolation, in the parameter $V$, has been found acceptable; for the gain, a quadratic interpolation was necessary. 
But for one pole and one zero, the interpolation was not so easy to realize. It was possible to locate them in precise areas but the path they followed is not exactly a straight line: in each case one point is out of a possible line. All these points have simply been excluded, and a linear interpolation adopted. Naturally, one can expect a consequence of this rough decision. Indeed it was visible on frequency responses of the interpolated controller evaluated for some value of $\hat{\theta}$ in $\Theta$. Figure 5 shows a typical bode response of one of the rough interpolated controller and of the LTI controller, for the same value of $\hat{\theta} \in \Theta$; they have the same aspect but there is a gap of almost $20 \mathrm{db}$ between the two magnitude curves. To decrease this gap, a new interpolation has been added such that, for the roll resonance frequency of the ship, the new magnitude is less or equal the magnitude of the corresponding LTI transfer function of the controller. The frequency responses of the final interpolated controllers are then correct (see figure 5 for one of these responses).

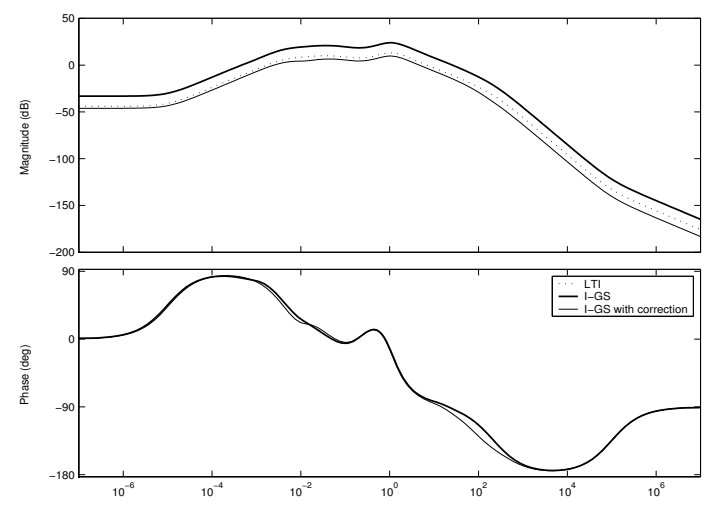

Fig. 5. Comparison of typical frequency responses.

\section{The final $\mathrm{H}_{\infty}-I-G S$ controller}

The final computed controller is composed of four transfer functions of the type

$$
K_{i j}(V, S Q F)(s)=g_{i j}(V, S Q F) \frac{n_{i j}(V, S Q F)(s)}{d_{i j}(V, S Q F)(s)}
$$

\section{Simulation RESUlts}

The following temporal simulations are performed with $\psi_{e}=90 \mathrm{deg}$ and a Sea State 5. Each controllers, invariant $\mathrm{H}_{\infty}$ LTI, $\mathrm{H}_{\infty}$-LPV-GS and $\mathrm{H}_{\infty}$-I-GS, have been discretized using the zero order hold method.

\section{A. $\mathrm{H}_{\infty}-I-G S$ versus invariant LTI $\mathrm{H}_{\infty}$ controllers}

Figures 6 and 7 illustrate the good behaviour of the $\mathrm{H}_{\infty}$ I-GS controller when the conditions vary and it is compared to some invariant $\mathrm{H}_{\infty}$ LTI controllers. Remember that $\phi$ is the roll angle, $\alpha$ and $\delta$ respectively define the position of the actuators (fins and rudders).

On figure 6, the SQF value is constant and equal to 8 whereas the speed vary from 15 to 25 knots. The LTI controller used was optimized for 15 knots and $\mathrm{SQF}=8$.

Between 200 and $250 \sec (V=15$ knots), the performances
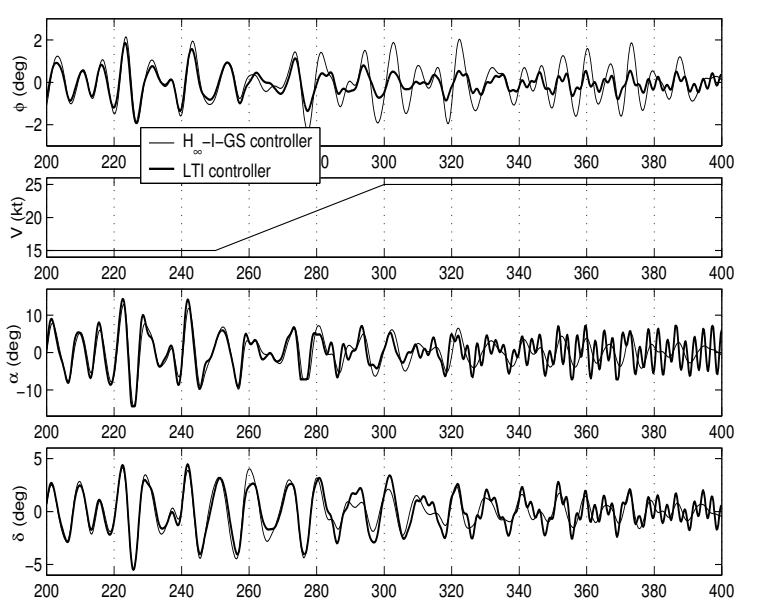

Fig. 6. $\mathrm{H}_{\infty}-\mathrm{I}-\mathrm{GS}$ and invariant $\mathrm{H}_{\infty}$ LTI controllers if $\mathrm{V}$ varies.

are very close to each other, the performances with the LTI controller, optimized for this case, are just a little bit better. Between 300 and $400 \sec (V=25$ knots), the roll attenuation seems to be better with the LTI controller! However, the agitation of the actuators, in this case shows that it is indeed unsuitable. On the contrary, the $\mathrm{H}_{\infty}$-I-GS controller keeps the same reduction $(\mathrm{SQF}=$ constant $=8)$ without degradation of the actuators activity: its adaptation to $V$ works.
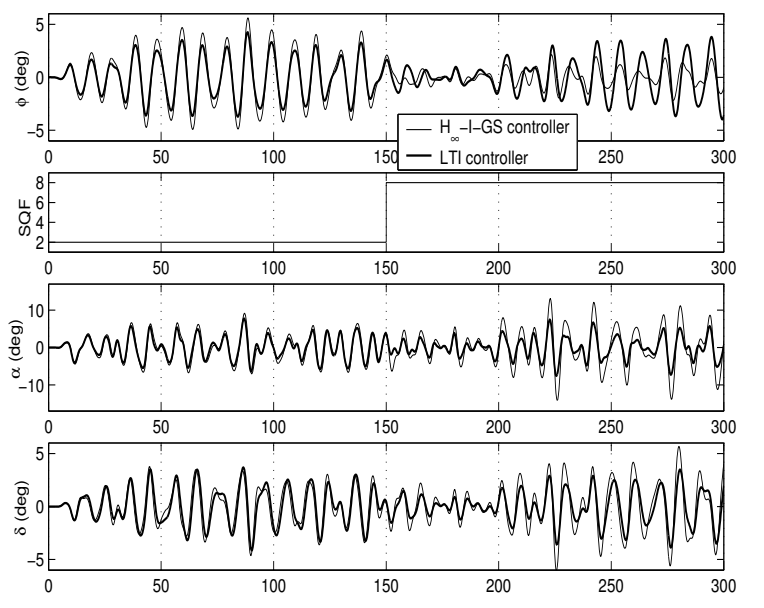

Fig. 7. $\mathrm{H}_{\infty}$-I-GS and invariant $\mathrm{H}_{\infty}$ LTI controllers if SQF varies.

On figure 7, the speed is constant and equal to 15 knots whereas the SQF parameter varies from 2 to 8 . Once more, between 0 and $150 \mathrm{sec}(S Q F=2)$, the LTI controller, optimized for $\mathrm{V}=15 \mathrm{kts}, \mathrm{SQF}=2$, is better than the $\mathrm{H}_{\infty}$-IGS one. However after $150 \mathrm{sec}(S Q F=8)$, the $\mathrm{H}_{\infty}$-IGS controller takes into account the new desired attenuation while the LTI one keeps the same previous performances.

Figure 6 and 7 showed the good performances of the $\mathrm{H}_{\infty}$-I-GS controller. To confirm the stability the closed loop and its performances properties, several statistics have been performed, including comparisons with the $\mathrm{H}_{\infty}$-LPV-GS controller. 


\section{B. $\mathrm{H}_{\infty}-I-G S$ versus $H_{\infty}-L P V-G S$ controllers}

Figure 8 shows the roll rate reduction (computed on 20 minutes long simulations) for different speeds, with $\mathrm{SQF}=8$, obtained with invariant $\mathrm{H}_{\infty}$ LTI controllers optimized at each considered speed, with the $\mathrm{H}_{\infty}$-I-GS controller and with the $\mathrm{H}_{\infty}$-LPV-GS controller.

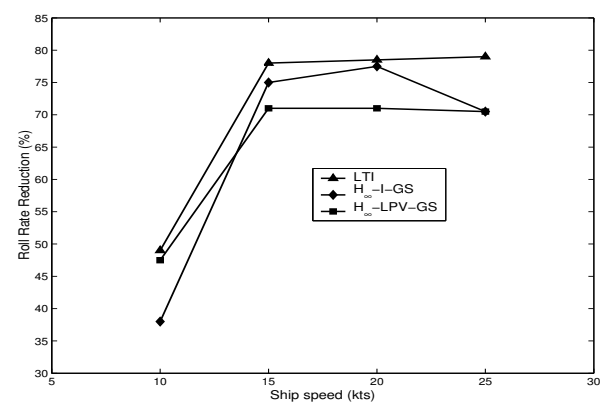

Fig. 8. Roll Rate Reduction $(S Q F=8)$.

Globally, for a given operating point, the performances obtained with the $\mathrm{H}_{\infty}$-I-GS controller are between those obtained by optimized invariant $\mathrm{H}_{\infty}$ LTI controllers and those obtained by the $\mathrm{H}_{\infty}$-LPV-GS controller.

More precisely, for 15 knots, the roll rate reduction of the $\mathrm{H}_{\infty}$-I-GS is worse than the one of the invariant $\mathrm{H}_{\infty}$ LTI; this confirms the simulation of figure 6 , between 200 and $240 \mathrm{sec}$. But it is better than in the $\mathrm{H}_{\infty}$-LPV-GS case; this can be seen on the left part of figure 9, (same scenario than in figure 6 , except that the LTI controller is replaced by the $\mathrm{H}_{\infty}$-LPV-GS one).

At 25 knots, figure 8 shows that the $\mathrm{H}_{\infty}$-I-GS and $\mathrm{H}_{\infty^{-}}$LPV-GS controllers have similar roll rate reduction; this is confirmed on figure 9 (right part), between 340 and $380 \mathrm{sec}$; Indeed the simulation shows almost no difference, except near $340 \mathrm{sec}$.

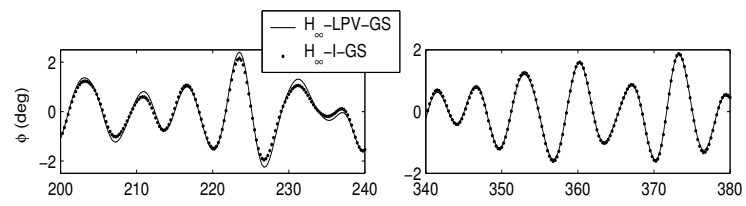

Fig. 9. $\mathrm{H}_{\infty}-\mathrm{I}-\mathrm{GS}, \mathrm{H}_{\infty}-\mathrm{LPV}-\mathrm{GS}(\mathrm{V}=15$ and $25 \mathrm{kts})$.

At 10 knots, the efficiency of the fins is reduced. This leads to a decrease in wished performances, and thus explains the global decrease in obtained performances. Moreover, the $\mathrm{H}_{\infty}$-I-GS controller was computed from LTI controllers which discrepancy induce imprecision in the eventual controller.

\section{Simulation conclusions}

The presented simulations correspond to particular conditions.
Other ones, not reported here [10] generalized the above observations: the $\mathrm{H}_{\infty}-\mathrm{I}-\mathrm{GS}$ controller seems to assure the closed loop stability (remember that there is no theoretical proof of it) and its performances are globally slightly better than the $\mathrm{H}_{\infty}$-LPV-GS performances.

\section{CONCLUSION}

The exposed study is an application of robust control techniques in marine system, namely the rudder/fin roll stabilisation of ship.

The aim of the paper was to synthetize a $\mathrm{H}_{\infty}$ interpolated Gain Scheduling controller, counter part of the $\mathrm{H}_{\infty}$ Linear Varying Parameter controller computed in [4], in order to test and compare the two approaches to obtain a Gain Scheduling controller.

Note that both controllers are based on the same data: the invariant standard models (for the $\mathrm{H}_{\infty}$-LPV-GS) or the associated $\mathrm{H}_{\infty}$ controllers (for the $\mathrm{H}_{\infty}-\mathrm{I}-\mathrm{GS}$ ). This probably explains why their performances are very close to each other. Considering the technical problems to obtain each controller it is difficult to show up one of the approaches. However it appears that the performances of the $\mathrm{H}_{\infty}$-I-GS controller are a little better which confirms the idea that some conservatism exists within the polytopic LPV approach.

\section{REFERENCES}

[1] A. Lloyd, Seakeeping, Ship Behaviour in Rough Weather, ser. Marine Technology. Hellis Horwood, 1989.

[2] M. Grimble, M. Katebi, and Y. Zhang, " $\mathrm{H}_{\infty}$ based ship fin-rudder roll stabilisation design," in 10th Ship Control Systems Symposium, vol. 5, Ottawa, Canada, 1993.

[3] M. Blanke, J. Adrian, K. Larsen, and J. Bentsen, "Rudder roll damping in coastal region sea conditions," in Proceedings of MCMC 2000, Aalgorg, Danemark, 2000.

[4] H. Tanguy and G. Lebret, "A gain scheduled control law for fin/rudder roll stabilisation of ships," in Proceedings of CAMS 2004, Ancona, Italy, July 2004.

[5] D. Leith and W. Leithead, "Survey of gain scheduling analysis and design," International Journal of Control, vol. 73, no. 11, pp. 1001$1025,2000$.

[6] W. Rugh and J. Shamma, "Research on gain scheduling," Automatica, vol. 36 , no. 10, pp. 1401-1425, 2000.

[7] R. Nichols, R. Reichert, and W. Rugh, "Gain scheduling for $\mathrm{H}_{\infty}$ controllers: A flight control example," IEEE transactions on Control System Technology, vol. 1, no. 2, pp. 69-78, 1993.

[8] A. Helmersson, "Methods for robust gain-scheduling," Ph.D. dissertation, Linkping University, Linkping, Sweden, 1995.

[9] J.-M. Biannic, "Commande robuste des systmes paramtres variables. applications en aronautique," Ph.D. dissertation, SUPAERO, Toulouse, France, 1996.

[10] H. Tanguy, "Synthèse de lois de commande à gains programmés pour la stabilisation en roulis des navires," Ph.D. dissertation, Université de Nantes, École Centrale de Nantes, Nantes, France, 2004.

[11] T. I. Fossen, Navigation and Guidance of Ocean Vehicles. New York: John Wiley \& sons, 1994.

[12] J. Sgobbo and M. Pearsons, "Rudder/fin roll stabilization of the uscg wmec 901 class vessel," Marine Technology, vol. 36, no. 3, pp. 157170 , july 1999 .

[13] H. Tanguy, G. Lebret, and O. Doucy, "Multi-objective optimisation of pid and $\mathrm{H}_{\infty}$ fin/rudder roll controller," in Proceedings of MCMC 2003, Girona, Spain, september 2003.

[14] D. Stiwell and W. Rugh, "Stability preserving interpolation methods for the synthesis of gain scheduled controllers," Automatica, vol. 36, no. 5, pp. 665-671, 2000. 